\title{
THE STRUCTURE OF A CRITICAL SET OF A COMPLETE INTERSECTION SINGULARITY
}

\author{
YOSEF YOMDIN
}

\begin{abstract}
Let $f:\left(C^{n}, 0\right) \rightarrow\left(C^{k}, 0\right)$ be the germ of an isolated complete intersection singularity. The structure of strata $\mu=$ const in a critical set of $f$ is studied. The main result is the following: if the dimension of the stratum $\mu=\mu(0)$ is $k-1$, then $f$ coincides with a family of hypersurface singularities with constant Milnor number.
\end{abstract}

1. We give here some definitions and results, concerning isolated complete intersection singularities (details can be found e.g., in [2]) and state the main theorem.

Let $f:\left(C^{n}, 0\right) \rightarrow\left(C^{k}, 0\right), n \geqslant k$, be the germ of a flat analytic mapping, $Y=f^{-1}(0)$, and let $Y$ have an isolated singular point at the origin. Such germs $f$ (and $Y$ ) will be called shortly SCI. The critical set $\Sigma(f)$ of $f$ is in this case $k-1$-dimensional and $f / \Sigma_{\text {red }}(f)$ is finite. $\left(\Sigma(f)\right.$ is considered with the structural ring $\theta_{\Sigma(f)}=\theta_{n} / J$, where $\theta_{n}$ is the ring of germs of analytic functions at $0 \in C^{n}$, and $J \subset \theta_{n}$ is the ideal, generated by all $(k \times k)$-minors of the Jacobian matrix of $f$.)

The Milnor number $\mu_{f}(0)$ of $f$ (or of $Y$ ) at $0 \in C^{n}$ is defined as the middle Betti number of the Milnor fiber $F=f^{-1}(\xi) \cap B_{\varepsilon}^{n}$. Here $B_{\varepsilon}^{n}$ is the $\varepsilon$-ball, centered at $0 \in C^{n}, \xi$ a regular value of $f, 1 \gg \varepsilon \gg|\xi|$.

Let $G_{k}^{l}$ be the Grassman manifold of $l$-dimensional subspaces of $C^{k}$. There is a Zariski open subset $U_{l} \subset G_{k}^{l}$, such that for $L \in U_{l}, f_{L}:\left(C^{n}, 0\right) \rightarrow C^{k} / L \cong\left(C^{k-l}, 0\right)$ is SCI, and the Milnor number of this singularity does not depend on $L \in U_{l}$. This number will be denoted by $\mu_{f}^{k-l}(0), \mu_{f}^{k}(0)=\mu_{f}(0)$.

Now let $f: M^{n} \rightarrow N^{k}, n \geqslant k$, be a flat mapping of regular complex manifolds, such that $f / \Sigma(f)$ is finite. Then for any $z \in M$ the germ of $f$ at $z$ is SCI and setting $\mu_{f}(z)$ to be equal to the Milnor number of this singularity, we define an integral function $\mu_{f}$ on $M$. The function $\mu_{f}$ is upper semicontinuous and $\mu_{f}(z)>0$ if and only if $z \in \Sigma(f)$.

Let $W_{\nu}(f)=\left\{z \in M \mid \mu_{f}(z) \geqslant \nu\right\}, \quad V_{\nu}(f)=\left\{z \in M \mid \mu_{f}(z)=\nu\right\}=W_{\nu}-W_{\nu+1}$. Then $W_{\nu}(f)$ are analytic subsets of $M, W_{0}(f)=M, W_{1}(f)=\Sigma(f)$, and if $M$ is compact, $W_{\nu}(f)=\varnothing$ for $\nu$ sufficiently big.

We consider also analytic subsets of $M, \sigma_{s}(f)=\{z \in M \mid \operatorname{rank} d f(z) \leqslant k-s\}$, $s=1, \ldots, k$. Clearly $\Sigma(f)=\sigma_{1}(f) \supseteq \cdots \supseteq \sigma_{k}$, and since $f / \Sigma(f)$ is finite, $\operatorname{dim} \sigma_{s} \leqslant$ $k-s$. (By [1, Corollary 1.7], if $z \in \sigma_{s}(f)$, then $\mu_{f}(z) \geqslant m(n, s)=\sum_{q=0}^{s-1} C_{n-s+q}^{n-s} 2^{q}$, and hence $\sigma_{s}(f) \subseteq W_{m(n, s)}(f)$.)

Received by the editors June 11, 1980 and, in revised form, June 25, 1981; presented to the Society at the Summer Institute on Singularities in Arcata, California, July 20-August 7, 1981.

1980 Mathematics Subject Classification. Primary 32B30; Secondary 14B05. 
Let $N^{\prime} \subset N$ be an analytic submanifold of $N$ such that $f$ is transversal to $N^{\prime}$, $M^{\prime}=f^{-1}\left(N^{\prime}\right), f^{\prime}=f \mid M^{\prime}: M^{\prime} \rightarrow N^{\prime}$. Then, clearly, $\mu_{f^{\prime}}=\mu_{f} \mid M^{\prime}, V_{\nu}\left(f^{\prime}\right)=V_{\nu}(f) \cap$ $M^{\prime}, \sigma_{s}\left(f^{\prime}\right)=\sigma_{s}(f) \cap M^{\prime}$.

The sign $f$ will be omitted below in notations of $\Sigma(f), \mu_{f}, V_{\nu}(f), W_{\nu}(f), \sigma_{s}(f)$.

In the local case of SCI $f:\left(C^{n}, 0\right) \rightarrow\left(C^{k}, 0\right), \mu, W_{\nu}, V_{\nu}, \sigma_{s}$ are germs at $0 \in C^{n}$. Note, that $V_{\mu(0)}=W_{\mu(0)}$ is an analytic germ.

The germ $f:\left(C^{n}, 0\right) \rightarrow\left(C^{k}, 0\right)$ is said to be equivalent to $g:\left(C^{n}, 0\right) \rightarrow\left(C^{k}, 0\right)$ if there exist germs of analytic diffeomorphisms $\varphi:\left(C^{n}, 0\right) \rightarrow\left(C^{n}, 0\right)$ and $\psi:\left(C^{k}, 0\right) \rightarrow$ $\left(C^{k}, 0\right)$, such that $\psi \circ f=g \circ \varphi$.

We say $f:\left(C^{n}, 0\right) \rightarrow\left(C^{k}, 0\right)$ is a family of hypersurface singularities with $\mu=$ const, if $f$ is equivalent to the germ $\Phi:\left(C^{k-1} \times C^{n-k+1}, 0\right) \rightarrow\left(C^{k-1} \times C, 0\right), \Phi(t, y)=$ $\left(t, h_{t}(y)\right)$, with $h_{t}:\left(C^{n-k+1}, 0\right) \rightarrow(C, 0)$ having for any $t$ an isolated singularity at $0 \in C^{n-k+1}$ with constant Milnor number.

Note, that for $n-k \neq 2$ a family of hypersurface singularities with $\mu=$ const is topologically trivial by [7].

TheOREM 1.1. Let $f:\left(C^{n}, 0\right) \rightarrow\left(C^{k}, 0\right)$ be $S C I$, and let $\operatorname{dim}_{0} V_{\mu(0)}=k-1$. Then $f$ is a family of hypersurface singularities with $\mu=\mu(0)$.

COROLLARY 1.2. If for $z \in \Sigma_{\text {red }}, \operatorname{dim}_{z} V_{\mu(z)}=k-1$, then in a neighborhood of $z$ $\Sigma_{\text {red }}=V_{\mu(z)}$ is smooth, and the restriction $f / \Sigma_{\text {red }}$ is regular at $z$. In particular, $z \in \Sigma_{\text {red }} \backslash \sigma_{2}$.

Originally, Theorem 1.1 was obtained under the additional restriction rank $d f(0)$ $\geqslant k-3$. The author would like to thank G. M. Greuel, who noted that a modification of arguments gives a proof of a present version of the theorem.

2. In this section we state some results of [8], concerning the global topology of strata $V_{\nu}$, which are used below.

Let $f: M^{n} \rightarrow N^{k}, \mu, V_{\nu}, W_{\nu}$ be as above, with $M$ and $N$ compact.

THEOREM 2.1 .

$$
\sum_{\nu \geqslant 1} \nu \cdot \chi\left(V_{\nu}\right)=\sum_{\nu \geqslant 1} \chi\left(W_{\nu}\right)=(-1)^{n-k}[\chi(\mathscr{F}) \cdot \chi(N)-\chi(M)]
$$

where $\chi$ denotes the topological Euler characteristic and $\mathcal{F}$ is a generic (nonsingular) fiber of $f$.

This theorem remains true under more weak conditions. We do not state here the general version, but give some corollaries, concerning the local structure of SCI.

Let $f:\left(C^{n}, 0\right) \rightarrow\left(C^{k}, 0\right)$ be a germ of SCI, $B_{\varepsilon}^{n}$ (resp. $\left.B_{\delta}^{k}\right)$ an open $\varepsilon$ (resp. $\delta$ )-ball, centered at $0 \in C^{n}$ (at $0 \in C^{k}$ ), $1 \gg \varepsilon \gg \delta>0$. Let $S$ be an analytic submanifold of $B_{\delta}^{k}$, such that $f$ is transversal to $S, Z=B_{\varepsilon}^{n} \cap f^{-1}(S), V_{\nu}^{\prime}=V_{\nu} \cap Z$. Theorem 2.1 holds for $f / Z: Z \rightarrow S$, and since the generic fiber $\mathscr{F}$ of $f / Z$ coincides with the Milnor fiber $F$ of $f, \chi(\mathcal{F})=1+(-1)^{n-k} \mu(0)$. Thus we have

Corollary 2.2 .

$$
\sum_{\nu=1}^{\mu(0)} \nu \cdot \chi\left(V_{\nu}^{\prime}\right)=\mu(0) \chi(S)+(-1)^{n-k}[\chi(S)-\chi(Z)] .
$$


Now let $L \in U_{l} \subset G_{k}^{l}$ be a $l$-dimensional subspace of $C^{k}, \xi \in C^{k} / L$ a sufficiently small regular value of $f_{L}:\left(C^{n}, 0\right) \rightarrow C^{k} / L$, and let $S$ be a parallel translation $L+\xi$ of $L$, intersected with $B_{\delta}^{k}, Z, V_{\nu}^{\prime}$ as above. In this case $\chi(S)=1, \chi(Z)=1+$ $(-1)^{n-k+l} \mu^{k-1}(0)$, and we obtain

Corollary 2.3 .

$$
\sum_{\nu=1}^{\mu(0)} \nu \cdot \chi\left(V_{\nu}^{\prime}\right)=\mu(0)+(-1)^{l+1} \mu^{k-1}(0)
$$

Finally, if we consider some small deformation $f^{\prime}$ of $f$ as the section of an appropriate family, then for corresponding strata $V_{\nu}^{\prime}$ of $f^{\prime}$ we obtain

Corollary 2.4 .

$$
\sum_{\nu=1}^{\mu(0)} \nu \cdot \chi\left(V_{\nu}^{\prime}\right)=\mu(0)
$$

3. In this section we prove Theorem 1.1 for singularities of codimension 2. Let $f$ : $\left(C^{n}, 0\right) \rightarrow\left(C^{k}, 0\right)$ be SCI. Let $\Sigma_{i}, i=1, \ldots, l$, be irreducible components of $\Sigma_{\text {red }}$ and let $k_{i}$ be the multiplicity of $\Sigma_{i}$ at 0 . For each $i$ the function $\mu$ is constant on a Zariski open subset of $\Sigma_{i}$ and its value on this subset we denote by $\mu_{i}$.

Lemma 3.1. Let rank $d f(0) \geqslant k-2$. Then

$$
\mu(0) \geqslant \sum_{i=1}^{l} k_{i} \mu_{i} .
$$

If rank $d f(0)=k-2$, then the strict inequality holds.

Proof. Let $L$ be a generic plane in $C^{k}$, passing through the origin. Then $Z=f^{-1}(L)$ is nonsingular, $\mu_{f / Z}=\mu_{f} / Z$ and $\Sigma(f / Z)=\Sigma(\cdot f) \cap Z$. Clearly, the multiplicity of $\Sigma_{i} \cap Z$ at 0 is greater than or equal to $k_{i}$, and hence it is sufficient to prove lemma for $f / Z: Z \rightarrow L$. Thus we can restrict ourself to the case $f:\left(C^{n}, 0\right) \rightarrow$ $\left(C^{2}, 0\right)$. Let $f$ be given by $w_{1}=f_{1}\left(z_{1}, \ldots, z_{n}\right), w_{2}=f_{2}\left(z_{1}, \ldots, z_{n}\right)$ in coordinates $z_{1}, \ldots, z_{n}$ in $C^{n}, w_{1}, w_{2}$ in $C^{2}$.

Performing a linear coordinate change in $C^{2}$ we can assume that $f_{1}$ has an isolated singularity at the origin. Then the Milnor number $\mu(0)$ can be computed by the following formula (see $[3,2])$ :

$$
\mu(0)=\operatorname{dim}_{C} \theta_{n} /\left\{f_{1}, J\right\}-\operatorname{dim}_{C} \theta_{n} / J_{1},
$$

where $J_{1}$ is the ideal, generated by $\partial f_{1} / \partial z_{1}, \ldots, \partial f_{1} / \partial z_{n}$. $\theta_{\Sigma}=\theta_{n} / J$ is a CohenMacaulay ring and $f_{1}$ is a parameter in $\theta_{\Sigma}$ (see e.g. [2]), hence

$$
\operatorname{dim}_{C} \theta_{n} /\left\{f_{1}, J\right\}=\left(\Sigma,\left\{f_{1}=0\right\}\right)_{0}=\sum_{z \in \Sigma \cap\left\{f_{1}=\delta\right\}}\left(\Sigma,\left\{f_{1}=\delta\right\}\right)_{z}
$$

for any small $\delta$, where $($,$) is the intersection multiplicity.$

Lemma 3.2. For $z \in \Sigma_{i} \backslash\{0\},\left(\Sigma,\left\{f_{1}=f_{1}(z)\right\}\right)_{z}=\mu_{i}$. 
Proof. This follows from the formula (2) applied at $z$, since $f_{1}$ is nonsingular at $z$ and then the second term in (2) is zero. (Clearly, the intersection multiplicity of $\Sigma$ with any regular hypersurface, transversal to $\Sigma$ at $z$ is also $\mu_{i}$.)

Thus, from (3) we have

$$
\operatorname{dim}_{C} \theta_{n} /\left\{f_{1}, J\right\}=\sum_{i=1}^{l} r_{i} \mu_{i},
$$

where $r_{i}=\left(\Sigma_{i},\left\{f_{1}=0\right\}\right)_{0}$.

From now on we assume that the coordinate system $z_{1}, \ldots, z_{n}$ in $C^{n}$ is chosen generically and in particular the following is true:

(1) $k_{i}=k_{i 1} \leqslant k_{i j}, i=1, \ldots, l, j=2, \ldots, n$, where $k_{i j}=\left(\Sigma_{i},\left\{z_{j}=0\right\}\right)_{0}$;

(2) $p_{i}=p_{i 1} \leqslant p_{i j}, i=1, \ldots, l, j=2, \ldots, n$, where $p_{i j}=\left(\Sigma_{i},\left\{\partial f_{1} / \partial z_{j}=0\right\}\right)_{0}$.

Since the singularity of $f_{1}$ at 0 is isolated, from (2) it follows that $p_{i}$ are finite, $i=1, \ldots, l$, i.e. $\partial f_{1} / \partial z_{1}$ is a parameter in $\theta_{\Sigma}$.

LEMMA 3.3. $r_{i} \geqslant k_{i}+p_{i}, i=1, \ldots, l$.

Proof. Let $n_{i}:(C, 0) \rightarrow\left(\Sigma_{i}, 0\right)$ be a normalization of $\Sigma_{i}$ and $\tau$ a coordinate in $C$. We have

$$
\begin{aligned}
z_{j} \circ n_{i} & =\alpha_{i j} \tau^{k_{i j}}+\ldots, \\
f_{1} \circ n_{i} & =\beta_{i} \tau^{r_{i}}+\ldots, \\
\partial f_{1} / \partial z_{j} \circ n_{i} & =\gamma_{i j} \tau^{p_{i j}}+\ldots,
\end{aligned}
$$

where $\alpha_{i j}, \beta_{i}, \gamma_{i j} \neq 0$. Then

$$
\begin{aligned}
\frac{d\left(f_{1} \circ n_{i}\right)}{d \tau} & =r_{i} \beta_{i} \tau^{r_{i}-1}+\ldots=\sum_{j=1}^{n} \frac{\partial f_{1}}{\partial z_{j}} \cdot \frac{d\left(z_{j} \circ n_{i}\right)}{d \tau} \\
& =\sum_{j=1}^{n}\left(\gamma_{i j} \tau^{p_{i j}}+\ldots\right) \cdot\left(\alpha_{i j} k_{i j} \tau^{k_{i j}-1}+\ldots\right) .
\end{aligned}
$$

By conditions (1) and (2) above the leading term on the right-hand side series is of degree $\geqslant k_{i}+p_{i}-1$ and since $r_{i} \neq 0\left(f_{1}(0)=0\right)$ it follows that $r_{i} \geqslant k_{i}+p_{i}$.

Now from (2), (4) and Lemma 3.3

$$
\mu(0) \geqslant \sum_{i=1}^{l} k_{i} \mu_{i}+\sum_{i=1}^{l} p_{i} \mu_{i}-\operatorname{dim}_{C} \theta_{n} / J_{1} .
$$

In turn, applying Lemma 3.2 and the fact that $\partial f_{1} / \partial z_{1}$ is a parameter in a Cohen-Macaulay ring $\theta_{\Sigma}$ we obtain

$$
\sum_{i=1}^{l} p_{i} \mu_{i}=\operatorname{dim}_{C} \theta_{n} /\left\{\partial f_{1} / \partial z_{1}, J\right\} .
$$

Clearly $\left\{\partial f_{1} / \partial z_{1}, J\right\} \subset J_{1}$, then $\sum_{i=1}^{\prime} p_{i} \mu_{i} \geqslant \operatorname{dim}_{C} \theta_{n} / J_{1}$ and hence

$$
\mu(0) \geqslant \sum_{i=1}^{l} k_{i} \mu_{i} \text {. }
$$


Now let rank $d f(0)=0$. Then $J \subset \mathfrak{m} J_{1}$, where $m$ is the maximal ideal of $\theta_{n}$. For corresponding ideals $\hat{J}, \hat{J}_{1}$ and $\hat{m}$ in the quotient ring $\theta_{n} /\left\{\partial f_{1} / \partial z_{1}\right\}$ also $\hat{J} \subset \hat{m} \hat{J}_{1}$, then by Nakayama lemma $\hat{J} \subset \hat{J}_{1}$ and hence $\left\{\partial f_{1} / \partial z_{1}, J\right\} \underset{\neq}{\subset J_{1}}$, which proves the strict inequality in (1).

Remark. Let $(Y, 0)$ be SCI, $f=\left(f_{1}, f_{2}\right):(Y, 0) \rightarrow\left(C^{2}, 0\right)$ a flat mapping, such that $f^{-1}(0)$ and $f_{1}^{-1}(0)$ also have isolated singularities at 0 . Lemmas 3.2 and 3.3 hold in this case and thus we have

$$
\mu_{f}(0)+\mu_{f_{1}}(0) \geqslant \sum_{i=1}^{l}\left(k_{i}+p_{i}\right) \mu_{i} .
$$

Proof of Theorem 1.1 For THE CASE rank $d f(0) \geqslant k-2$. If $\operatorname{dim}_{0} V_{\mu}(0)=k-1$, then at least one of $\mu_{i}$ is equal to $\mu(0)$. From Lemma 3.1 it follows immediately that $l=1, k_{1}=1, \mu_{1}=\mu(0)$ and rank $d f(0)=k-1$. In other words, $\Sigma_{\text {red }}$ is nonsingular and $\mu$ is constant on it. Now for a generic line $\lambda$ in $C^{k}, Y^{\prime}=f^{-1}(\lambda)$ is nonsingular and $\left(\Sigma_{\text {red }}, Y^{\prime}\right)_{0}=1$ (for otherwise a hypersurface singularity $f / Y^{\prime}$ can be split into more than one singularity with the same Milnor number). Hence $f / \Sigma_{\text {red }}$ is regular. By the implicit function theorem we can choose coordinates $t_{1}, \ldots, t_{k-1}, z_{1}, \ldots, z_{n-k+1}$ in $C^{n}$ and $t_{1}^{\prime}, \ldots, t_{k-1}^{\prime}, w$ on $C^{k}$ such that $\Sigma_{\text {red }}$ is defined by $z_{1}=\cdots=z_{n-k+1}=0$, and $t_{i}=t_{i}^{\prime} \circ f, i=1, \ldots, k-1$, i.e., $f$ is equivalent to $\Phi:\left(C^{k-1} \times C^{n-k+1}, 0\right) \rightarrow$ $\left(C^{k-1} \times C, 0\right), \Phi(t, z)=\left(t, h_{t}(z)\right)$.

4. In this section we complete the proof of Theorem 1.1. Let $f:\left(C^{n}, 0\right) \rightarrow\left(C^{k}, 0\right)$ be SCI, and let $\operatorname{dim}_{0} V_{\mu(0)}=k-1$. Then one of irreducible components of $\Sigma_{\text {red }}$, say $\Sigma_{1}$, is contained in $V_{\mu(0)}$. We shall prove, that $\Sigma_{\text {red }}=\Sigma_{1}$. Indeed, assume that $\Sigma_{2}$ is another component of $\Sigma_{\text {red }}$.

Choose a plane $L \in U_{2} \subset G_{k}^{2}$ in $C^{k}$ and let as in Corollary $2.3, \xi$ be a sufficiently small regular value of $f_{L}:\left(C^{n}, 0\right) \rightarrow C^{k} / L, L_{\xi}=L+\xi, S=L_{\xi} \cap B_{\delta}^{k}, Z=f^{-1}(S) \cap$ $B_{\varepsilon}^{n}, f^{\prime}=f / Z, \Sigma^{\prime}=\Sigma \cap Z$. Let $\omega_{1}=Z \cap \Sigma_{1}, \omega_{2}=Z \cap \Sigma_{2}$. Since $\mu_{f^{\prime}}=\mu_{f} / Z$ is constant on $\omega_{1}$, we see (by already proved part of Theorem 1.1) that the germ of $\Sigma^{\prime}$ at any point $z \in \omega_{1}$ coincides with $\omega_{1}$. In particular, $\omega_{1} \cap \omega_{2}=\varnothing$.

Now $f\left(\Sigma_{1}\right)$ and $f\left(\Sigma_{2}\right)$ are two hypersurfaces in $C^{k}$ and their intersection $Q$ is at least $(k-2)$-dimensional. Then $S \cap Q \neq \varnothing$ ( $L$ is chosen generically). Take some $t_{0} \in S \cap Q$. We see, that $f^{-1}\left(t_{0}\right) \cap \Sigma$ contains at least two different points $z_{1} \in \omega_{1}$ and $z_{2} \in \omega_{2}$ with $\mu\left(z_{1}\right)=\mu(0), \mu\left(z_{2}\right)>0$. But this contradicts to the following inequality:

If for $t \in C^{k}, f^{-1}(t) \cap \Sigma=\left\{z_{1}, \ldots, z_{q}\right\}$, then $\sum_{i=1}^{q} \mu\left(z_{q}\right) \leqslant \mu(0)$. (Proof: let an analytic set $\left\{z_{1}, \ldots, z_{q}\right\}$ be defined by equations $h_{1}=0, \ldots, h_{s}=0$. Considering the Morse function $\varphi=\left|h_{1}\right|^{2}+\cdots+\left|h_{s}\right|^{2}$ on $f^{-1}\left(t^{\prime}\right)$, where $t^{\prime}$ is a regular value of $f$ near $t$, we see, that the Milnor fiber $f^{-1}\left(t^{\prime}\right)$ can be obtained from the union of Milnor fibers of $f$ at $z_{1}, \ldots, z_{q}$, by attaching cells of dimensions $\leqslant m-k$.)

Thus, $\Sigma_{\text {red }}$ coincides with $V_{\mu(0)}$ and is irreducible.

Now, let us prove, that rank $d f(0)=k-1$. Indeed, assume, that $r=\operatorname{rank} d f(0)<$ $k-1$. Choose a coordinate system $w_{1}, \ldots, w_{k}$ in $C^{k}$, such that

(a) rank $d(\pi \circ f)(0)=r$, where $\pi: C^{k} \rightarrow C^{r}, \pi\left(w_{1}, \ldots, w_{k}\right)=\left(w_{1}, \ldots, w_{r}\right)$,

(b) a generic hyperplane $L$ in $C^{k-r}=\left\{\left(0, \ldots, 0, w_{r+1}, \ldots, w_{k}\right)\right\}$, considered as a $k-r-1$ dimensional subspace in $C^{k}$, belongs to $U_{k-r-1} \subset G_{k}^{k-r-1}$. 
Now choose coordinates $z_{1}, \ldots, z_{n}$ in $C^{n}$ such that $f\left(z_{1}, \ldots, z_{n}\right)=\left(w_{1}, \ldots, w_{k}\right)=$ $\left(z_{1}, \ldots, z_{r}, f_{r+1}, \ldots, f_{k}\right)$. Let $h=\left(f_{r+1}, \ldots, f_{k}\right) / C^{n-r}: C^{n-r} \rightarrow C^{k-r}$. We have rank $d h(0)=0$, and $k-r \geqslant 2$.

We apply to $f$ Corollary 2.3 , taking $L$ with $\operatorname{dim} L=k-r-1$ to be a hyperplane in $C^{k-r}$ (according to (b)). Since $\Sigma_{\text {red }}=V_{\mu(0)}, V_{\nu}^{\prime}=\varnothing$ for $\nu \neq \mu(0)$ and Corollary 2.3 gives

$$
\begin{gathered}
\mu(0) \chi\left(V_{\mu(0)}^{\prime}\right)=\mu(0)+(-1)^{k-r} \mu^{r+1}(0), \text { or } \\
\mu(0)\left[\chi\left(V_{\mu(0)}^{\prime}\right)-1\right]=(-1)^{k-r} \mu^{r+1}(0) .
\end{gathered}
$$

By the construction, we have $\mu(0)=\mu_{f}(0)=\mu_{h}(0) ; \mu^{r+1}(0)=\mu_{f}^{r+1}(0)=\mu_{h}^{1}(0)$, and by $[1$, Corollary 1.6$]$, we obtain

$$
\mu(0)>\mu^{r+1}(0) \text {. }
$$

Now, (4.1) and (4.2) lead to a contradiction: if $\chi\left(V_{\mu(0)}^{\prime}\right)=1$, by $(4.1), \mu^{r+1}(0)=0$ and hence rank $d f(0) \geqslant r+1$, which contradicts the assumption. If $\chi\left(V_{\mu(0)}^{\prime}\right) \geqslant 2$, by (4.1), $k-r$ must be even and $\mu^{r+1}(0) \geqslant \mu(0)$, which contradicts (4.2). Finally, if $\chi\left(V_{\mu(0)}^{\prime}\right) \leqslant 0$, then by $(4.1), k-r$ is odd and once more $\mu^{r+1}(0) \geqslant \mu(0)$. This contradiction proves that rank $d f(0)=k-1$ and in this case Theorem 1.1 was already proved in $\$ 3$.

REMARK. Corollary 1.2 shows, that $f$ is topologically equisingular along the strata $\mu=$ const of the maximal dimension.

If the dimension of stratum $\mu=$ const is strictly less than $k-1$, this is not true, even for rank $d f=k-1$ (see [5]). rank $d f$ can also change in such deformations (see [6, Example 4.6.3]). However, the following kind-of-topological triviality can be proved by method of [4]: in a family of SCI with $\mu=$ const (and $n-k \neq 2$ ) the Milnor fiber and the knot $Y \cap S_{\varepsilon}^{n-1}$, where $S_{\varepsilon}^{n-1}=\partial B_{\varepsilon}^{n}$, do not change their topological type.

\section{REFERENCES}

1. M. Guisti and J. P. Henry, Minorations de nombres de Milnor, Bull. Soc. Math. France 108 (1980), 17-45.

2. G. M. Greuel, Der Gauss-Manin-Zusammenhang isolierter Singularitaten von vollstandigen Durchschnitten, Math. Ann. 214 (1975), 235-266.

3. Le Dung Trang, Calculation of the Milnor number of isolated singularity of complete intersection, Functional Anal. Appl. 8 (1974), 127-131.

4. Le Dung Trang and C. P. Ramanujam, The invariance of Milnor's number implies the invariance of topological type, Amer. J. Math. 98 (1976), 67-78.

5. F. Pham, Remarques sur l'equisingularité universelle, preprint, Université de Nice, 1971.

6. B. Teissier, The hunting of invariants in the geometry of discriminants, Real and Complex Singularities, Proceedings of the Nordic Summer School/NAVF, Oslo, 1976.

7. J. G. Timourian, The invariance of Milnor's number implies topological triviality, Amer. J. Math. 99 (1977), 437-446.

8. Y. Yomdin, Euler characteristics of strata $\mu=$ const for isolated singularities of complete intersections, preprint, Ben Gurion University of the Negev, 1979.

Department of Mathematics, Ben Gurion University of THE Negev, BeER-SheVa 84120, ISRAel 